TITLE:

\title{
Berry's Phase and Euclidean Path Integral
}

$\operatorname{AUTHOR}(S):$

柏, 太郎; 二摩, 修司; 迫田, 誠治

CITATION:

柏, 太郎 ... [et al]. Berry's Phase and Euclidean Path Integral. 数理解析研 究所講究録 1994, 869: 172-181

ISSUE DATE:

1994-05

URL:

http://hdl.handle.net/2433/83986

RIGHT: 


\title{
Berry's Phase and Euclidean Path Integral
}

\author{
柏 太郎 ( Taro Kashiwa) \\ 九大理 二摩修司 (Shuji Nima) \\ 迫田誠治 (Seiji Sakoda)
}

\section{Introduction}

'84 年のベリーの論文 [1] 以来ベリーの位相については、その幾何学的な性質が注目さ れ、非常に多くの研究がなされてきた $[2,3]$ 。この背景にはゲージ理論の成功によって、接 続の微分幾何に対する関心の高まりがあったものと言えそうだが、すこしそうした幾何学 的な側面ばかりが強調されすぎたようにも感じられる。もともと、べリーの位相は断熱近 似に基づいて導出されたものであり、そこにはそうした近似が許されるという力学的な事 情があったはずである。この近似によって得られる結果の面白さは否定されるものではない が、くまでも近似の中での話である。いわゆる、速い自由度と遅い自由度というような アイデアには、まさしくそのような力学的な仮定が含まれている。このような事情を量子 力学的に考えるには経路積分の方法が力を発揮するはずであるが、これまで経路積分の手 法を用いてべリーの位相を解析するという仕事が見られなかった。そこでわれわれは、ま ず量子力学における断熱近似を経路積分の中にいかに取り入れるかを調べ、その枠組みの 中でベリーの位相をとらえる方法を考える。次のステップとして経路積分の特長を活かす ために Euclid 化について議論する。次の二節では、こうしてできた方法を、よく知られた 二つの例に適用してみる。最後にここまでのまとめと、今後の課題について触れたい。

\section{2 経路積分における断熱近似}

時間に依存する Hamiltonian $H(\mathbf{X}(t)) （ \mathbf{X}(t)$ はいくつかの parameter で時間 $t$ の関数） に対して、 $\mathbf{X}(t)=\mathbf{X}_{0}$ のときの固有值、固有べクトルを

$$
\begin{gathered}
H\left(\mathbf{X}_{0}\right)\left|n\left(\mathbf{X}_{0}\right)\right\rangle=E_{n}\left(\mathbf{X}_{0}\right)\left|n\left(\mathbf{X}_{0}\right)\right\rangle \\
\left\langle n\left(\mathbf{X}_{0}\right) \mid m\left(\mathbf{X}_{0}\right)\right\rangle=\delta_{n, m}, \\
\sum_{n}\left|n\left(\mathbf{X}_{0}\right)\right\rangle\left\langle n\left(\mathbf{X}_{0}\right)\right|=\mathbf{1}
\end{gathered}
$$


とする。この系の Schrödinger 方程式

$$
i \hbar \frac{\partial}{\partial t}|\Psi(t)\rangle=H(\mathbf{X}(t))|\Psi(t)\rangle
$$

は、 $|\Psi(t)\rangle=U(t)|\Psi(0)\rangle$ によって導入される時間発展演算子 $U(t)$ に対して

$$
i \hbar \frac{\partial}{\partial t} U(t)=H(\mathbf{X}(t)) U(t), \quad U(0)=\mathbf{1}
$$

となり、この解は形式的には

$$
\begin{aligned}
U(T)= & \lim _{N \rightarrow \infty}\left(1-\frac{i}{\hbar} \Delta t H\left(\mathbf{X}\left(t_{N-1}\right)\right)\right)\left(1-\frac{i}{\hbar} \Delta t H\left(\mathbf{X}\left(t_{N-2}\right)\right)\right) \\
& \times \cdots \times\left(1-\frac{i}{\hbar} \Delta t H\left(\mathbf{X}\left(t_{1}\right)\right)\right)\left(1-\frac{i}{\hbar} \Delta t H\left(\mathbf{X}\left(t_{0}\right)\right)\right) \\
\equiv & \mathbf{T} \exp \left(-\frac{i}{\hbar} \int_{0}^{T} d t H(\mathbf{X}(t))\right)
\end{aligned}
$$

によって与えられる。ここで $\Delta t=T / N, t_{k}=k \Delta t(k=0, \cdots, N), t_{0}=0, t_{N}=T$ などの記号 を用いた。通常、断熱近似と呼ばれるのは、

$$
\langle n(\mathbf{X}(t)) \mid m(\mathbf{X}(t-\Delta t))\rangle \approx \delta_{n, m}\left(1-\Delta t\left\langle n(\mathbf{X}(t))\left|\frac{\partial}{\partial t}\right| n(\mathbf{X}(t))\right\rangle\right)
$$

を仮定して

$$
U(T)_{n, m} \approx \delta_{n, m} \exp \left[\int_{0}^{T} d t\left(-\frac{i}{\hbar} E_{n}(\mathbf{X}(t))-\left\langle n(\mathbf{X}(t))\left|\frac{\partial}{\partial t}\right| n(\mathbf{X}(t))\right\rangle\right)\right]
$$

としてしまうものだが、これではこの近似を超えてコレクションを考慮するにはまったく不 便であるし、経路積分に持ち込む方針が見えない。そこでもう少していねいにやるために

$$
\begin{array}{ccc}
V^{\dagger}(t) H(t) V(t) & = & H^{V}(t) \\
H^{V}(t) & =\sum_{n} E_{n}(t)|n(\mathbf{X}(0))\rangle\langle n(\mathbf{X}(0))|
\end{array}
$$

となるようなユニタリー演算子 $V(t)$ を考える。 $V(t)$ は一意的には決まらないがその不定性 を適当な条件で除いてやれば

$$
V^{\dagger}(t)|n(t)\rangle=e^{-i \gamma_{n}(t)}|n(0)\rangle
$$

となるようにできる。ここで $\gamma_{n}(t)$ は

$$
\begin{aligned}
\gamma_{n}(t) & =i \int_{0}^{t} d t^{\prime}\left\langle n\left(t^{\prime}\right)\left|\frac{\partial}{\partial t^{\prime}}\right| n\left(t^{\prime}\right)\right\rangle \\
& =i \int_{\mathbf{X}(0)}^{\mathbf{X}(t)} d X^{a}\left\langle n(\mathbf{X})\left|\frac{\partial}{\partial X^{a}}\right| n(\mathbf{X})\right\rangle
\end{aligned}
$$


で与えらる。(2.6)に $V^{\dagger}(t) V(t)=\mathbf{1}$ と完全性関係 (2.3) とを繰り返し挿入することにより

$$
\begin{aligned}
U(T)= & \lim _{N \rightarrow \infty} \sum_{\substack{\left\{n_{k}\right\} \\
k=0, \cdots, N}} V\left(t_{N}\right)\left|n_{N}(0)\right\rangle\left\langle n_{N}(0)\right| \\
& \times\left(1-\frac{i}{\hbar} \Delta t\left\{H^{V}\left(t_{N-1}\right)-i \hbar V^{\dagger}\left(t_{N-1}\right) \dot{V}\left(t_{N-1}\right)\right\}\right)\left|n_{N-1}(0)\right\rangle\left\langle n_{N-1}(0)\right| \\
& \times \cdots \times\left(1-\frac{i}{\hbar} \Delta t\left\{H^{V}\left(t_{0}\right)-i \hbar V^{\dagger}\left(t_{0}\right) \dot{V}\left(t_{0}\right)\right\}\right)\left|n_{0}(0)\right\rangle\left\langle n_{0}(0)\right|
\end{aligned}
$$

これはさらに

$$
\begin{aligned}
U(T)= & \lim _{N \rightarrow \infty} \sum_{\left\{n_{k}\right\}} V\left(t_{N}\right)\left|n_{N}(0)\right\rangle\left\langle n_{N}(0)\left|\left(1-\frac{i}{\hbar} \Delta t H^{V}\left(t_{N-1}\right)\right)\right| n_{N-1}(0)\right\rangle \\
& \times\left\langle n_{N-1}(0)\left|\times \cdots \times\left(1-\frac{i}{\hbar} \Delta t H^{V}\left(t_{0}\right)\right)\right| n_{0}(0)\right\rangle\left\langle n_{0}(0)\right|\{1+O(1 / T)\} \\
= & \sum_{n} \exp \left(-\frac{i}{\hbar} \int_{0}^{T} d t E_{n}(t)+i \gamma_{n}(T)\right)|n(T)\rangle\langle n(0)|\{1+O(1 / T)\}
\end{aligned}
$$

と変形できるから、Feynman 核 $K\left(q, q^{\prime} ; T\right)=\left\langle q|U(T)| q^{\prime}\right\rangle$ に対する断熱近似は

$$
\begin{aligned}
K\left(q, q^{\prime} ; T\right)= & \sum_{n} \psi_{n}(q ; \mathbf{X}(T)) \psi_{n}^{*}\left(q^{\prime} ; \mathbf{X}(0)\right) \exp \left(-\frac{i}{\hbar} \int_{0}^{T} d t E_{n}(t)+i \gamma_{n}(T)\right) \\
& \times\{1+O(1 / T)\}
\end{aligned}
$$

の $O(1 / T)$ の寄与を無視することだとわかる。ここで $\psi_{n}(q ; \mathbf{X}(t))=\langle q \mid n(\mathbf{X}(t))\rangle$ は $H(\mathbf{X}(t))$

の『瞬間的な』固有関数である。一方、(2.14)の左辺は経路積分表示

$$
\begin{aligned}
K\left(q, q^{\prime} ; T\right) & \lim _{N \rightarrow \infty} \int \prod_{i=1}^{N} \frac{d p(i)}{2 \pi \hbar} \prod_{j=1}^{N-1} d q(j) \\
\times & \left.\exp \left(\frac{i}{\hbar} \sum_{k=1}^{N}\{p(k) \Delta q(k)-\Delta t H(p(k), \bar{q}(k) ; \mathbf{X}(k))\}\right)\right|_{q_{N}=q, q_{0}=q^{\prime}}, \\
\Delta q(k) & \equiv q(k)-q(k-1), \\
\bar{q}(k) & \equiv \frac{1}{2}(q(k)+q(k-1))
\end{aligned}
$$

を持つはずだから、この経路積分を $1 / T$ の leading で評価すれば断熱近似を行ったことに なる。以上は、一般的だがまったく形式的な話でこれでは経路積分の特長が活かされない。 一般の Hamiltonian に対して (2.15) の積分を㛜密に遂行し(2.14)の形を見出すことは事実 上不可能で、なんらかの (たとえばWKB) 近似を用いることで基底状態の構造を知ること ができれば良いという場合がほとんどである。このような観点から $(2.15)$ そのものでなく Euclid 化した経路積分を考察すべきであろうと思われる。次の節では、われわれの問題意 識を明らかにするとともにこの Euclid 経路積分についてさらに考えてみる。 


\section{Euclid 化と問題点}

ベリーの位相と言えば時間依存性を持った Hamiltonian が出発点になるのが通常の approach であるが、その力学的な効果を考察の対象とするならば、むしろ閉じた系から出発 するのが自然である。そのなかで、力学的な事情によって effective に外場のように扱える 部分系とそれを通じて時間依存性を持った Hamiltonian で記述される部分系とに分離でき る様な場合が、これまでによく議論されてきた場合に当たると考えたい。たとえば、質量 のスケールが極端に異なる 2 つの部分系が結合している場合、次のような議論ができるで あろう。

出発点としては、

$$
\hat{H}=H(\hat{\mathbf{P}}, \hat{\mathbf{Q}})+h(\hat{p}, \hat{q} ; \hat{\mathbf{Q}})
$$

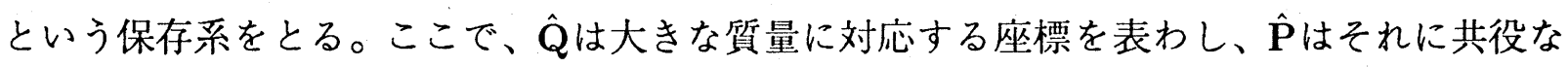

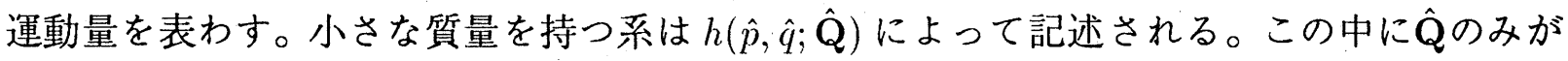
現われているのは、 $\hat{\mathbf{P}}$ との結合があると以下に述べるような近似がうまく機能しないから である。 $P, Q$ 系の質量が十分大きいときこの系全体に対する Feynman 核は、

$$
\begin{aligned}
& K\left(\mathbf{Q}, q ; \mathbf{Q}^{\prime}, q^{\prime} ; T\right) \equiv\left\langle\mathbf{Q}, q\left|\exp \left(-\frac{i}{\hbar} T \hat{H}\right)\right| \mathbf{Q}^{\prime}, q^{\prime}\right\rangle \\
& =\int \mathcal{D} \mathbf{P} \mathcal{D} \mathbf{Q} \mathcal{D} p \mathcal{D} q \exp \left(\frac{i}{\hbar} \int_{0}^{T} d t\left\{\mathbf{P} \frac{d \mathbf{Q}}{d t}+p \frac{d q}{d t}-H(\mathbf{P}, \mathbf{Q})-h(p, q ; \mathbf{Q})\right\}\right) \\
& \underset{M \rightarrow \infty}{\simeq} K_{\text {heavy }}\left(\mathbf{Q}, \mathbf{Q}^{\prime} ; T\right) K_{\mathbf{Q}_{c}}\left(q, q^{\prime} ; T\right)
\end{aligned}
$$

のような分離した形を持つことが期待される。ここで、 $K_{h \in a v y}\left(\mathbf{Q}, \mathbf{Q}^{\prime} ; T\right)$ は重いほうの系の 古典解のまわりで評価した Feynman 核で、 $K_{\mathbf{Q}_{c}}\left(q, q^{\prime} ; T\right)$ のほうは、この古典解の影響下で の軽い系に対する Fenyman 核である。具体的には、

$$
\begin{aligned}
K_{\mathbf{Q}_{c}}\left(q, q^{\prime} ; T\right) & \equiv \int \mathcal{D} p \mathcal{D} q \exp \left(\frac{i}{\hbar} \int_{0}^{T} d t\left\{p \frac{d q}{d t}-h_{0}(p, q ; \mathbf{X})-V\left(p, q ; \mathbf{Q}_{c}\right)\right\}\right) \\
h_{0}(p, q ; \mathbf{X}) & \equiv \frac{1}{2}\left\{Z(t) p^{2}+2 Y(t) p q+X(t) q^{2}\right\}
\end{aligned}
$$

という一般的な形を持つ。ただし、次の節で考える一般化された調和振動子を記述する部 分を $h_{0}(p, q ; \mathbf{X})$ として 3 次以上の項と分けて書いた。このようにして $K_{\mathbf{Q}_{c}}\left(q, q^{\prime} ; T\right)$ から得 られた軽い系に対する effective な時間依存性を持った Hamiltonian は一般にベリーの位相 を与えると考えられるが、それは上で考えた極限的な場合に得られるものであるから、も ともとの $(3.1)$ には含まれていなかったものである。したがって、いったん得られたべリー の位相が近似を上げることによってどのように消滅していくのかということはたいへん興 味深い問題である。このあたりについてもっと詳しく調べたいと考えているのだが、ここ ではとりあえずこの最低次でべリーの位相を見出す方法について議論する。 
通常の Euclid 化の処方 $t \rightarrow-i t$ に従えば(2.4)を

$$
-\hbar \frac{\partial}{\partial t}|\Psi(t)\rangle=H(\hat{p}, \hat{q} ; \mathbf{X}(-i t))|\Psi(t)\rangle
$$

と変更するところであるが、これでは右辺の Hamiltonian の構造がまったく違う系になっ てしまう。そこで、(3.4) はやめて

$$
-\hbar \frac{\partial}{\partial t}|\Psi(t)\rangle=H(\hat{p}, \hat{q} ; \mathbf{X}(t))|\Psi(t)\rangle
$$

をとる。これは、上で考えたような系の $K_{\mathbf{Q}_{c}}\left(q, q^{\prime} ; T\right)$ に現われる重い系の影響下での軽い 系の基底状態を議論するのにふさわしい方法である。この(3.5)に対して (2.5) と同様にし て Euclid 版の時間発展演算子 $\tilde{U}(T)$ を導入すれば (2.13) に対応して

$$
\begin{aligned}
\tilde{U}(T)= & \lim _{N \rightarrow \infty}\left(1-\frac{1}{\hbar} \Delta t \hat{H}\left(t_{N-1}\right)\right) \times \cdots \times\left(1-\frac{1}{\hbar} \Delta t \hat{H}\left(t_{0}\right)\right) \\
= & \sum_{n} \exp \left(-\frac{1}{\hbar} \int_{0}^{T} d t E_{n}(\mathbf{X}(t))+i \gamma_{n}(T)\right)|n(T)\rangle\langle n(0)| \\
& \times\{1+O(1 / T)\}
\end{aligned}
$$

が得られる。したがって、Feynman 核も(2.14)に対応して

$$
\begin{aligned}
\tilde{K}\left(q, q^{\prime} ; T\right) \equiv & \left\langle q|\tilde{U}(T)| q^{\prime}\right\rangle \\
= & \sum_{n} \psi_{n}(q ; \mathbf{X}(T)) \psi_{n}^{*}\left(q^{\prime} ; \mathbf{X}(0)\right) \exp \left(-\frac{1}{\hbar} \int_{0}^{T} d t E_{n}(\mathbf{X}(t))+i \gamma_{n}(T)\right) \\
& \times\{1+O(1 / T)\}
\end{aligned}
$$

が得られ、これはまた

$$
\begin{aligned}
\tilde{K}\left(q, q^{\prime} ; T\right)= & \lim _{N \rightarrow \infty} \int \prod_{i=1}^{N} \frac{d p(i)}{2 \pi \hbar} \prod_{j=1}^{N-1} d q(j) \\
& \times\left.\exp \left(\frac{1}{\hbar} \sum_{k=1}^{N}\{i p(k) \Delta q(k)-\Delta t H(p(k), \bar{q}(k) ; \mathbf{X}(k))\}\right)\right|_{q_{N}=q, q_{0}=q^{\prime}}
\end{aligned}
$$

という経路積分表示を持ち $T \rightarrow \infty$ の極限で

$$
(3.8) \underset{T \rightarrow \infty}{\simeq} \psi_{0}(q ; \mathbf{X}(T)) \psi_{0}^{*}\left(q^{\prime} ; \mathbf{X}(0)\right) \exp \left(-\frac{1}{\hbar} \int_{0}^{T} d t E_{0}(\mathbf{X}(t))+i \gamma_{0}(T)\right)
$$

が導かれ基底状態に関する情報を取り出してくれる。ここで $\exp (\cdots)$ の肩の中で虚数部分 はベリーの位相を与えている。以上の議論をまとめると、ベリーの位相を取り出す我々の 処方は次のようになる。

(i) 与えられた Hamiltonian に対する Euclid 版 Feynman 核を考える。ただし、その際 Hamiltonian に含まれる parameters の時間依存性はそのままにしておく。 
(ii) Euclid 版 Feynman 核の $T \rightarrow \infty$ 極限を調べる。

(iii) 指数関数の虚数部分で $1 / T$ について $O(1)$ の量を拾い出す。

これによってベリーの位相だけでなくHamiltonianの『瞬間的」基底状態に対する固有值、波 動関数も求められる。もし必要ならば、(3.9)をもとの (3.8) から引き去ったものを Feynman 核とみてこの操作を繰り返せば、第 1 励起状態、第 2 励起状態、‥というふうに情報を引 き出すことができる。この処方がきちんと機能することを確かめるための例題として、次 の節では一般化された調和振動子を、またその次の節では磁場中の spin の例を考察する。

\section{4 一般化された調和振動子}

『一般化された調和振動子』の Hamiltonian は

$$
H(\hat{p}, \hat{q} ; \mathbf{X}(t))=\frac{1}{2}\left\{Z(t) \hat{p}^{2}+Y(t)(\hat{p} \hat{q}+\hat{q} \hat{p})+X(t) \hat{q}^{2}\right\}
$$

で与えられる。ここで、 $X(t) Z(t)_{Y}^{2}(t)>0, Z(t)>0$ を仮定しておく。この Hamiltonianに 対する Euclid 版 Feynman 核は

$$
\begin{aligned}
& \tilde{K}\left(q, q^{\prime} ; T\right) \equiv\left\langle q\left|\mathbf{T} \exp \left(-\frac{1}{\hbar} \int_{0}^{T} d t H(\hat{p}, \hat{q} ; \mathbf{X}(t))\right)\right| q^{\prime}\right\rangle=\int \mathcal{D} p \mathcal{D} q \\
& \times\left.\exp \left(\frac{1}{\hbar} \int_{0}^{T} d t\left\{i p \frac{d q}{d t}-\frac{1}{2}\left[Z(t) p^{2}+2 Y(t) p q+X(t) q^{2}\right]\right\}\right)\right|_{q(0)=q^{\prime}, q(T)=q}
\end{aligned}
$$

で与えられるが、これに

$$
\left(\begin{array}{c}
q(t) \\
p(t)
\end{array}\right) \longmapsto\left(\begin{array}{c}
Q(t) \\
P(t)
\end{array}\right)=\left(\begin{array}{c}
\frac{q(t)}{\sqrt{Z}(t)} \\
\sqrt{Z(t) p} p(t)+\frac{\alpha(t) q(t)}{\sqrt{Z}(t)}
\end{array}\right)
$$

という変数変換を施すと

$$
\begin{aligned}
\tilde{K}\left(q, q^{\prime} ; T\right) & = \\
\Phi\left(q, q^{\prime}\right) & \times \int \mathcal{D} P \mathcal{D} Q \exp \left(\frac{1}{\hbar} \int_{0}^{T} d t\left\{i P \frac{d Q}{d t}-\frac{1}{2}\left(P^{2}+\Omega^{2}(t) Q^{2}\right)\right\}\right)
\end{aligned}
$$

となる。ただし、

$$
\begin{gathered}
\alpha(t) \equiv Y(t)-\frac{i}{2} \frac{\dot{Z}(t)}{Z(t)} \quad, \quad \dot{Z}(t) \equiv \frac{d Z(t)}{d t} \\
\Omega^{2}(t) \equiv \omega^{2}(t)-\omega_{1}(t), \\
\omega^{2}(t)=X(t) Z(t)-Y^{2}(t), \quad \omega_{1}(t)=-i Z(t) \frac{d}{d t}\left(\frac{\alpha}{Z}\right)(t),
\end{gathered}
$$




$$
\Phi\left(q, q^{\prime}\right) \equiv \exp \left(-\frac{i}{2 \hbar}\left\{\frac{\alpha(T)}{Z(T)} q^{2}-\frac{\alpha(0)}{Z(0)} q^{\prime 2}\right\}\right),
$$

で$\Phi\left(q, q^{\prime}\right)$ は波動関数に効く部分を表わす。 $P$ 積分の後 $Q$ を古典解 $Q_{c}$ の周りで展開すると (4.4) は

$$
\begin{aligned}
\tilde{K}\left(q, q^{\prime} ; T\right) & =\frac{1}{\sqrt{2 \pi \hbar \Delta(T)}} \exp \left(\frac{-1}{\hbar} S_{c}\left(q, q^{\prime} ; T\right)\right) \\
S_{c}\left(q, q^{\prime} ; T\right) & \equiv \frac{i}{2}\left\{\frac{\alpha(T)}{Z(T)} q^{2}-\frac{\alpha(0)}{Z(0)} q^{\prime 2}\right\}+\frac{1}{2}\left[Q_{c} \frac{d Q_{c}}{d t}\right]_{0}^{T} \\
\frac{1}{\sqrt{2 \pi \hbar \Delta(T)}} & =\int \mathcal{D} \xi \exp \left(-\frac{1}{\hbar} \int_{0}^{T} d t \frac{1}{2} \xi\left(-\frac{d^{2}}{d t^{2}}+\Omega^{2}(t)\right) \xi\right)
\end{aligned}
$$

となる。ただし、 $\xi$ 積分は $\xi(0)=\xi(T)=0$ という境界条件の下で行う。 $\Delta(T) や S_{c}\left(q, q^{\prime} ; T\right)$ は古典解 $Q_{c}$ が求まれば計算できる。1/Tに関してここで必要な精度での古典解は

$$
\begin{aligned}
Q_{c}(s)= & \frac{1}{\sqrt{\omega(s)} \sinh \Theta(1)}\left\{\sqrt{\frac{\omega(1)}{Z(1)}} q \sinh \Theta(s)+\sqrt{\frac{\omega(0)}{Z(0)}} q^{\prime} \sinh \bar{\Theta}(s)\right\} \\
& \times\{1+O(1 / T)\}, \\
\Theta(s)= & T \int_{0}^{s} d \sigma\left(\omega(\sigma)+\frac{1}{2 T} \frac{\omega_{1}(\sigma)}{\omega(\sigma)}\right) \\
= & T \int_{0}^{s} d \sigma\left\{\omega(\sigma)-\frac{i}{2 T} \frac{Z(\sigma)}{\omega(\sigma)} \frac{d}{d \sigma}\left(\frac{Y(\sigma)}{Z(\sigma)}\right)\right\}, \\
\bar{\Theta}(s)= & \Theta(1)-\Theta(s)
\end{aligned}
$$

で与えられる。ただし、1/Tを見やすくするために $s=t / T を$ 用いた。(4.10)をもとに (4.9a) は、

$$
\begin{aligned}
\tilde{K}\left(q, q^{\prime} ; T\right)= & \frac{1}{\sqrt{2 \pi \hbar \sinh \Theta(T)}}\left(\frac{\omega(T) \omega(0)}{Z(T) Z(0)}\right)^{\frac{1}{4}} \exp \left(\frac{-1}{2 \hbar \sinh \Theta(T)} \sqrt{\frac{\omega(T) \omega(0)}{Z(T) Z(0)}}\right. \\
\times & \left\{\left(\sqrt{\frac{Z(0) \omega(T)}{Z(T) \omega(0)}} q^{2}+\sqrt{\frac{Z(T) \omega(0)}{Z(0) \omega(T)}} q^{\prime 2}\right) \cosh \Theta(T)-2 q q^{\prime}\right\} \\
& \left.-\frac{i}{2 \hbar}\left(\frac{Y(T)}{Z(T)} q^{2}-\frac{Y(0)}{Z(0)} q^{\prime 2}\right)\right)
\end{aligned}
$$

と求められ、 $T \rightarrow \infty$ とすると

$$
\begin{aligned}
& \tilde{K}\left(q, q^{\prime} ; T\right) \quad \underset{T \rightarrow \infty}{\simeq}\left(\frac{\omega(T)}{\pi \hbar Z(T)}\right)^{\frac{1}{4}}\left(\frac{\omega(0)}{\pi \hbar Z(0)}\right)^{\frac{1}{4}} e^{-\frac{1}{2} \Theta(T)} \\
& \times \exp \left(-\frac{1}{2 \hbar Z(T)}(\omega(T)+i Y(T)) q^{2}-\frac{1}{2 \hbar Z(0)}(\omega(0)-i Y(0)) q^{\prime 2}\right)
\end{aligned}
$$


が得られる。これから、基底状態について、ベリーの位相、『エネルギー固有值』、波動関 数をそれぞれ次のように読み取ることができる。

$$
\begin{gathered}
\gamma_{0}(T)=\frac{1}{4} \int_{0}^{T} d t \frac{Z(t)}{\omega(t)} \frac{d}{d t}\left(\frac{Y(t)}{Z(t)}\right)=\frac{1}{4} \int_{\mathbf{X}(0)}^{\mathbf{X}(T)} d X^{a} \frac{Z}{\sqrt{X Z-Y^{2}}} \frac{\partial}{\partial X^{a}}\left(\frac{Y}{Z}\right), \\
E_{0}(\mathbf{X}(t))=\frac{\hbar}{2} \omega(\mathbf{X}(t)) \\
\psi_{0}(q ; \mathbf{X}(t))=\left(\frac{\omega(t)}{\pi \hbar Z(t)}\right)^{\frac{1}{4}} \exp \left(-\frac{1}{2 \hbar Z(t)}(\omega(t)+i Y(t)) q^{2}\right)
\end{gathered}
$$

\section{5 磁場中の spin}

ここでは、生成・消滅演算子で spin を表現しそれらに対する coherent state を用いて経 路積分を構成する方法で磁場中の spin の例を調べる。

二種類の生成・消滅演算子

$$
\left[a_{i}, a_{j}^{\dagger}\right]=\delta_{i, j}, \quad\left[a_{i}, a_{j}\right]=\left[a_{i}^{\dagger}, a_{j}^{\dagger}\right]=0 \quad(i, j=1,2)
$$

とPauli 行列を用いて

$$
J_{\alpha}=\frac{\hbar}{2} \mathbf{a}^{\dagger} \sigma_{\alpha} \mathbf{a}, \quad S=\frac{1}{2} \mathbf{a}^{\dagger} \mathbf{a}
$$

を定義して、Hamiltonian

$$
H=-\mathbf{B}(t) \cdot \mathbf{J}
$$

を考える。ここで $\mathbf{B}(t)$ は外部磁場

$$
\mathbf{B}(t) \equiv B(t) \mathbf{n}(t)=B(t)(\sin \theta(t) \cos \phi(t), \sin \theta(t) \sin \phi(t), \cos \theta(t)) ; \quad B(t)>0
$$

を表わす。(5.3) は、射影演算子

$$
P_{J}=\int_{0}^{2 \pi} \frac{d \lambda}{2 \pi} \exp \left(i \lambda\left(\mathbf{a}^{\dagger} \mathbf{a}-2 J\right)\right)
$$

を掛けることにより磁場中の $\operatorname{spin}(大 き さ ~ J)$ を記述する。 $H, P_{J}$ が生成・消滅演算子で表わ されているので、コヒーレント状態

$$
a_{i}|\mathbf{z}\rangle=z_{i}|\mathbf{z}\rangle, \quad\left\langle\mathbf{z} \mid \mathbf{z}^{\prime}\right\rangle=\exp \left(\mathbf{z}^{\dagger} \mathbf{z}^{\prime}\right) \quad, \int \frac{d \mathbf{z}^{\dagger} d \mathbf{z}}{\pi^{2}}|\mathbf{z}\rangle e^{-\mathbf{z}^{\dagger} \mathbf{z}}\langle\mathbf{z}|=\mathbf{1}
$$

を用いた Euclid 版 Feynman 核の経路積分表示を spin Jのセクターに対して

$$
\begin{aligned}
& \tilde{K}^{(J)}\left(\mathbf{z}, \mathbf{z}^{\prime} ; T\right) \\
& =\lim _{N \rightarrow \infty} \int_{0}^{2 \pi} \frac{d \lambda}{2 \pi}\left\langle\mathbf{z}\left|\left(1-\frac{1}{\hbar} \Delta t H\left(t_{N-1}\right)\right) \cdots\left(1-\frac{1}{\hbar} \Delta t H\left(t_{0}\right)\right) e^{i \lambda\left(\mathbf{a}^{\dagger} \mathbf{a}-2 J\right)}\right| \mathbf{z}^{\prime}\right\rangle \\
& =\int_{0}^{2 \pi} \frac{d \lambda}{2 \pi} \exp \left(\mathbf{z}^{\dagger} R(T) \mathbf{z}^{\prime} e^{i \lambda}-2 i J \lambda\right) \\
& =\frac{1}{(2 J) !}\left(\mathbf{z}^{\dagger} R(T) \mathbf{z}^{\prime}\right)^{2 J}
\end{aligned}
$$


のように構成できる。ここで $R(T)$ は

$$
R(T)=\left(\begin{array}{cc}
e^{\Theta(T)} \cos \frac{\theta}{2} \cos \frac{\theta^{\prime}}{2} & e^{\Theta(T)-i \phi^{\prime}} \cos \frac{\theta}{2} \sin \frac{\theta^{\prime}}{2} \\
+e^{-\Theta(T)-i\left(\phi-\phi^{\prime}\right)} \sin \frac{\theta}{2} \sin \frac{\theta^{\prime}}{2} & -e^{-\Theta(T)-i \phi} \sin \frac{\theta}{2} \cos \frac{\theta^{\prime}}{2} \\
e^{\Theta(T)+i \phi} \sin \frac{\theta}{2} \cos \frac{\theta^{\prime}}{2} & e^{\Theta(T)+i\left(\phi-\phi^{\prime}\right)} \sin \frac{\theta}{2} \sin \frac{\theta^{\prime}}{2} \\
-e^{-\Theta(T)+i \phi^{\prime}} \cos \frac{\theta}{2} \sin \frac{\theta^{\prime}}{2} & +e^{-\Theta(T)} \cos \frac{\theta}{2} \cos \frac{\theta^{\prime}}{2}
\end{array}\right),
$$

で与えられ、また $\Theta(T)$ は

$$
\Theta(T)=\frac{T}{2} \int_{0}^{1} d \tau\left\{B(\tau)-\frac{i}{T}(1-\cos \theta(\tau)) \frac{d \phi}{d \tau}(\tau)\right\}
$$

で、 $\theta, \theta^{\prime}, \phi, \phi^{\prime}$ は磁場の初期および最終の方向を表わす。もとの (5.3) に対する Euclid 経路

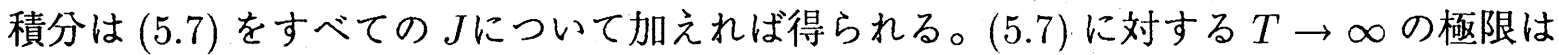

$$
\begin{aligned}
\tilde{K}^{(J)} & \left(\mathbf{z}, \mathbf{z}^{\prime} ; T\right) \\
& \underset{T \rightarrow \infty}{\simeq} e^{2 J \Theta(T)} \frac{1}{(2 J) !}\left(z_{1}^{*} \cos \frac{\theta}{2}+z_{2}^{*} e^{i \phi} \sin \frac{\theta}{2}\right)^{2 J}\left(z_{1}^{\prime} \cos \frac{\theta^{\prime}}{2}+z_{2}^{\prime} e^{-i \phi^{\prime}} \sin \frac{\theta^{\prime}}{2}\right)^{2 J} \\
& =\psi_{0}^{J}(\mathbf{z} ; \mathbf{B}(T)) \psi_{0}^{J^{*}}\left(\mathbf{z}^{\prime} ; \mathbf{B}(0)\right) \exp \left(J T \int_{0}^{1} d \tau\left\{B(\tau)-\frac{i}{T}(1-\cos \theta(\tau)) \frac{d \phi}{d s}(\tau)\right\}\right)
\end{aligned}
$$

となるので、この場合にもベリーの位相、『エネルギー固有值』、波動関数が各 $\operatorname{spin} J セ ク$ ターごとの基底状態に対して

$$
\begin{gathered}
\gamma_{0}=-J \int_{0}^{T} d t(1-\cos \theta(t)) \frac{d \phi}{d t}(t)=-J \int_{\phi^{\prime}}^{\phi} d \phi(1-\cos \theta), \\
E_{0}(\mathbf{B}(t))=-\hbar J B(t), \\
\psi_{0}^{J}(\mathbf{z} ; \mathbf{B}(t))=\frac{1}{\sqrt{(2 J) !}}\left(z_{1}^{*} \cos \frac{\theta(t)}{2}+z_{2}^{*} e^{i \phi(t)} \sin \frac{\theta(t)}{2}\right)^{2 J}
\end{gathered}
$$

というふうに読み取れる。

\section{6 まとめ}

経路積分で断熱近似を取り扱い、ベリーの位相を見出す方法を考察した。これによって、 ベリーの位相だけでなく Hamiltonian の固有值や対応する波動関数も求められる通常とは 異なる Euclid 化の方法、(i) - (iii)、が有効であることを、一般化された調和振動子および 磁場中の spinの例で確かめた。特に (i) の処方についていうと、通常のように (3.4)を使え 
ないのは、それが重い系の古典的な運動の影響下で軽い系が運動するという状況をうまく 反映しないためであるが、その意味を十分に調べて見るべきである。これは今後の研究課題 としたい。また、その中でベリ一の位相の力学的な性質という側面にも触れて行くことに なると考えている。紹介した例は簡単なものであるが、計算の詳細についてはかなりの省 略をした。経路積分のより厳密な取り扱いなどは我々の論文 [4]を参照していただきたい。

\section{参考文献}

[1] M. V. Berry, Proc. R. London, A392(1984), 45.

[2] A. Shapere and F. Wilczek (Eds), "Geometric Phases in Physics", World Scientific, Singapore, 1989.

[3] Y. Aharonov and J. Anandan, Phys. Rev. Lett. 58(1987), 1593.

[4] T. Kashiwa, S. Nima and S. Sakoda, Ann. Phys. 220(1992), 248 